\title{
Film and Line Tension Effects on the Attachment of Particles to an Interface
}

\author{
II. Shapes of the Bubble (Drop) and the External Meniscus
}

\author{
PETER ATANASSOV KRALCHEVSKY, IVAN BOYANOV IVANOV, \\ AND ALEXANDER DUSHKOV NIKOLOV \\ Laboratory of Thermodynamics and Physico-Chemical Hydrodynamics, Faculty of Chemistry, \\ University of Sofia, 1126 Sofia, Bulgaria
}

Received April 23, 1984; accepted August 15, 1985

\begin{abstract}
By using the method of matched asymptotic expansions analytical equations for the shapes of a sessile (pendant) bubble or drop and the external meniscus around it are derived. The results are compared with the numerical solutions of S. Hartland and R. W. Hartley, for the bubble or drop ("Axisymmetric Fluid-Liquid Interfaces," Elsevier, Amsterdam, 1976), and C. Huh and L. E. Scriven, for the external meniscus [J. Colloid Interface Sci. 30, 323 (1969)]. When the capillary number is small, the analytical solutions are in excellent agreement with the computer calculations. (c) 1986 Academic Press, Inc.
\end{abstract}

\section{INTRODUCTION}

It was already pointed out in Part $\mathrm{I}^{2}$ that the determination of the film and line tensions requires knowledge of the angles $\varphi_{c}$ and $\psi_{c}$ (for definitions see Figs. 1 and 2 below) at which the surfaces of the bubble (drop) and the external meniscus meet the horizontal plane. These angles are not amenable to direct experimental determination but can be calculated from the measured values of some other geometric characteristics of the system (more specifically, the radius of the contact line $r_{\mathrm{c}}$ and the radius of the bubble at its equator $R$ ) and the solutions of the Laplace equations of the two surfaces. Such numerical solutions are now available $(4,5)$, but they are not entirely appropriate for our needs. They have a relatively large step with respect to the angles $\left[5^{\circ}\right.$ in (5)] and do not cover the range $\psi_{\mathrm{c}}<0.5^{\circ}$ whereas we require a precision of the angle calculation of the order of $0.01 \%$, and values of $\psi_{\mathrm{c}}$ as small as $0.1^{\circ}$ are still of importance

\footnotetext{
${ }^{1}$ Author to whom correspondence should be addressed.

${ }^{2}$ The other papers in this series are quoted hereafter as Part I (1), Part III (2), and Part IV (3).
}

in the calculation of the differential interference pattern (see Part III). In addition, the equation of the external meniscus surface is calculated in (4) with a precision lower than needed for the theoretical evaluation of the observed differential interference pattern. Last but not least, calculation of $\varphi_{c}$ and $\psi_{c}$ is only an intermediate step toward our final goalcalculation of the film and line tensions (see Part IV). Had we used computer results at this stage, we would have had to use much more complicated computer programs for the final calculations with the ensuing decrease in the precision of the results. We also believe that the relatively simple analytical expressions for the shapes of the bubble (drop) and the external meniscus derived here can be useful for other purposes as well, e.g., for measurement of the surface tension by the methods of sessile or pendant drop where exact knowledge of the shape of the surface is of crucial importance to the precision of the final result (6).

We will be concerned here only with very small particles (of radius smaller than say 1 $\mathrm{mm}$ ). Then the capillary number is usually much smaller than unity and it can serve as a 
small parameter, so that the method of matched asymptotic expansions $(7,8)$ can be used to derive the equations of the surfaces of interest. Since, however, solutions of the Laplace equation for the bubble (drop) surface and the external meniscus are carried out in quite different ways, these two particular problems will be dealt with in different sections, Sections 2 and 3, respectively. In both cases the results will also be valid for a system in a centrifugal field, provided that the gravity acceleration is replaced by $\omega R_{0}^{2}$, where $\omega$ is the angular velocity and $R_{0}$ is the distance between the mass center of the particle and the axis of rotation. With minor alterations the method described in Section 2 can be applied to derive the shape of a pendant drop as well. Although we will skip the details, the initial equations and the final results (Eqs. [1], [13]-[16], and [22]) will be written so that they could be used for this system as well. The precision and range of validity of our analytical solutions are checked against the numerical results of Huh and Scriven (4) and Hartland and Hartley (5) in Section 4. Calculation of the shapes of the bubble (drop) and the external meniscus is treated in the present paper as two separate problems, that is, why $\psi_{c}$ in Eqs. [73] and [74] is looked at as an independent parameter. In fact, both $\varphi_{\mathrm{c}}$ and $\psi_{\mathrm{c}}$ can be calculated from the experimentally measured values of $r_{\mathrm{c}}$ and $R$ and the equations derived in the present paper. The procedure to be followed to achieve these results is explained in Part IV.

\section{PERTURBATIONAL EQUATIONS FOR THE SHAPE OF SMALL BUBBLES AND DROPS}

Having in mind our purpose, in this section we will focus our attention mainly on a bubble attached to a fluid interface (Fig. 1A). The results are also valid, however, for a bubble pressed against a solid wall and a drop lying on a fluid or solid interface (Fig. 1B). The equations obtained describe not only the surface existing in the real system, but also its extrapolation as shown in Fig. 1. The shapes

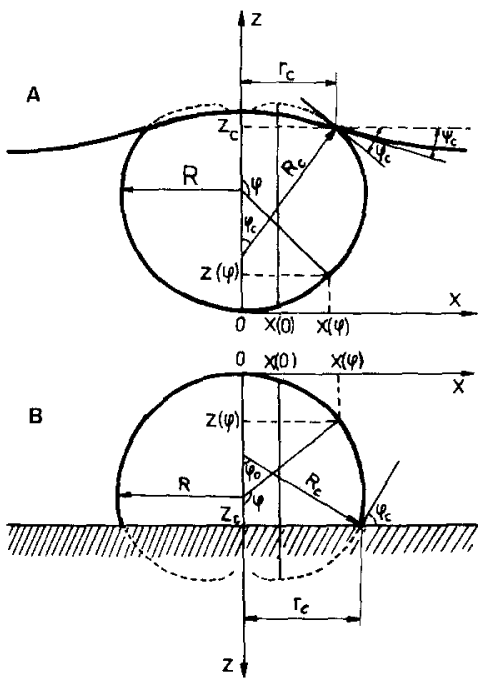

FIG. 1. A fluid particle at an interface: (A) fluid interface, (B) solid interface. $R$ and $R_{\mathrm{c}}$ are the radii of curvature of the generatrix of the bubble surface at its equator and at the contact line, $\varphi$ is the running slope angle and $\varphi_{\mathrm{c}}$ is its value at the contact line, whose radius and distance from zero of the coordinate system are denoted by $r_{\mathrm{c}}$ and $z_{\mathrm{c}}$. The extrapolated bubble surface is depicted by a dashed line and $x(0)$ is the abscissa of the extremum of $z$ at $\varphi=0$.

of these systems, $z(x)$, obey the Laplace equation, which can also be written in the parametric form ${ }^{3}(9)$

$$
\begin{aligned}
\frac{1}{x} \frac{d}{d x}(x \sin \varphi) & =\frac{2}{b} \pm \frac{\Delta \rho g}{\sigma} z, \\
\frac{d z}{d x} & =-\operatorname{tg} \varphi
\end{aligned}
$$

where $\varphi$ is the angle between the normal to the curve $z(x)$ and the positive direction of the $z$ axis, $b$ is the radius of curvature at $z=0$, $\Delta \rho$ is the density difference between the heavier and the lighter phases, $\sigma$ is surface tension, and $g$ is gravity acceleration. By introducing the dimensionless variables

$$
\bar{x}=x / b, \quad \bar{z}=z / b, \quad \beta=\Delta \rho g b^{2} / \sigma,
$$

\footnotetext{
${ }^{3}$ The upper sign refers to a sessile interface and the lower one, to a pendant interface.
} 
we have

$$
\begin{aligned}
\frac{1}{\bar{x}} \frac{d}{d \bar{x}}(\bar{x} \sin \varphi) & =2+\beta \bar{z}, \\
\frac{d \bar{z}}{d \varphi} & =-\operatorname{tg} \varphi \frac{\mathrm{d} \bar{x}}{\mathrm{~d} \varphi},
\end{aligned}
$$

which must be solved along with the boundary conditions

$$
\bar{x}=0, \quad \bar{z}=0 \quad \text { at } \quad \varphi=180^{\circ} .
$$

The solution of [3] for $\beta=0$ leads to a spherical shape, i.e., to

$$
\bar{x}_{0}=\sin \varphi, \quad \bar{z}_{0}=1+\cos \varphi .
$$

Therefore, for small $\beta$ we seek a solution of the form ${ }^{4}$

$$
\begin{aligned}
& \bar{x}(\varphi, \beta)=\sin \varphi+\sum_{n \geqslant 1} \beta^{n} \bar{x}_{n}(\varphi), \\
& \bar{z}(\varphi, \beta)=1+\cos \varphi+\sum_{n \geqslant 1} \beta^{n} \bar{z}_{n}(\varphi) .
\end{aligned}
$$

By substituting [6] in [3] and equating the coefficients before each power of $\beta$ we obtain systems of equations for $\bar{x}_{n}(\varphi)$ and $\bar{z}_{n}(\varphi)$

$$
\begin{gathered}
\frac{1}{\cos \varphi} \frac{d \bar{x}_{n}}{d \varphi}+\frac{\bar{x}_{n}}{\sin \varphi}=W_{n}(\varphi), \\
\frac{d \bar{z}_{n}}{d \varphi}=-\operatorname{tg} \varphi \frac{\mathrm{d} \bar{x}_{n}}{\mathrm{~d} \varphi}, \quad n=1,2, \ldots,
\end{gathered}
$$

where (the prime denotes differentiation with respect to $\varphi$ )

$$
\begin{aligned}
& W_{1}(\varphi)=-(1+\cos \varphi)=-\bar{z}_{0}(\varphi), \\
& W_{2}(\varphi)=\left(\frac{\bar{x}_{1}^{\prime}}{\cos \varphi}\right)^{2}+\left(\frac{\bar{x}_{1}}{\sin \varphi}\right)^{2}-\bar{z}_{1}(\varphi),
\end{aligned}
$$

etc. The solution of [7], subjected to the boundary conditions

\footnotetext{
${ }^{4}$ In principle $\sigma$ is a function of $b$ via the Tolman-Buff $(10,11)$ equation $\sigma=\sigma_{\infty}\left(1+2 \delta_{\infty} / b\right)$, where $\delta_{\infty}$ is the distance between the equimolecular dividing surface and the surface of tension. If this expression for $\sigma$ is substituted in [2] the analytical result will remain the same and the contribution of the curvature effect due to $\sigma$ will be of the order of $2 \delta_{\infty} / b$, which for our experiments is negligible.
}

$$
\bar{x}_{n}\left(\varphi=180^{\circ}\right)=0, \quad \bar{z}_{n}\left(\varphi=180^{\circ}\right)=0,
$$

reads

$$
n=1,2, \ldots \text {, }
$$

$$
\bar{x}_{1}(\varphi)=\frac{1}{3} \operatorname{ctg} \frac{\varphi}{2}-\frac{1}{6} \sin 2 \varphi-\frac{1}{2} \sin \varphi,
$$

$\bar{x}_{2}(\varphi)$

$$
\begin{gathered}
=\left(\frac{3}{4}+\frac{1}{2} \cos \varphi-\frac{2}{9} \sin ^{2} \varphi-\frac{1}{3} \ln \left(\sin \frac{\varphi}{2}\right)\right) \\
\times \sin \varphi-\frac{1}{2}\left(1+\frac{1}{9} \operatorname{ctg}^{2} \frac{\varphi}{2}\right) \operatorname{ctg} \frac{\varphi}{2} \\
\bar{z}_{1}(\varphi)=\frac{1}{3} \sin ^{2} \varphi+\frac{2}{3} \ln \left(\sin \frac{\varphi}{2}\right) \\
-\frac{1}{2}(1+\cos \varphi),
\end{gathered}
$$

so that the final solution can be put in the form (see footnote 3 )

$$
\begin{aligned}
& \bar{x}(\varphi)= \sin \varphi \pm \beta \bar{x}_{1}(\varphi) \\
&+\beta^{2} \bar{x}_{2}(\varphi)+O\left(\beta^{3} \bar{x}_{3}(\varphi)\right), \\
& \bar{z}(\varphi)=1+\cos \varphi \pm \beta \bar{z}_{1}(\varphi)+O\left(\beta^{2} \bar{z}_{2}(\varphi)\right) .
\end{aligned}
$$

The error involved in Eqs. [13] and [14] as a function of the system parameters is analyzed in Section 4.

The values of $\bar{x}$ and $\bar{z}$ at the equator, i.e., at $\varphi=90^{\circ}$, is of importance for the measurement of surface tension by the sessile and pendant drop methods. From [13] and [14] these are (see footnote 3 )

$\bar{x}\left(90^{\circ}\right)=1 \mp \frac{\beta}{6}+\frac{\beta^{2}}{6}\left(\ln 2-\frac{1}{6}\right)+\cdots$

$$
\bar{z}\left(90^{\circ}\right)=1 \mp \frac{\beta}{6}(1+\ln 4)+\cdots \cdot
$$

An alternative approach for solving [3] is to eliminate $\bar{z}$ by differentiating the first equation with respect to $x$ and substituting in the result $d \bar{z} / d \bar{x}$ from the second equation. For $90^{\circ} \leqq \varphi \leqq 180^{\circ}$ the result is 


$$
\frac{d}{d \bar{x}}\left(\frac{1}{\bar{x}} \frac{d(\bar{x} S)}{d \bar{x}}\right)=\beta \frac{S}{\sqrt{1-S^{2}}},
$$

where $S$ stands for $\sin \varphi$. The boundary conditions that we will use are

$S=0 \quad$ and $\quad \frac{1}{\bar{x}} \frac{d(\bar{x} S)}{d \bar{x}}=2$ at $\quad \bar{x}=0$

The second condition states that the curvature at $\bar{x}=\bar{z}=0$ must be $2 / b$. The result of the solution is (see footnote 3 )

$$
\begin{aligned}
S(\bar{x}) & \equiv \sin \varphi \\
& =\bar{x} \pm \beta S_{1}(\bar{x})+\beta^{2} S_{2}(\bar{x})+\cdots,
\end{aligned}
$$

with

$$
\begin{aligned}
& S_{1}(\bar{x})=\frac{\bar{x}}{6 v}\left[v-2(v-1)^{2}\right], \\
& S_{2}(\bar{x})=\frac{\bar{x}}{6}\left(\frac{2}{v}-\ln \frac{2}{v}-1\right),
\end{aligned}
$$

where

$$
v=1+\sqrt{1-\bar{x}^{2}}
$$

In some cases Eq. [19] is more convenient than Eq. [13] because it yields directly $\varphi$ as a function of $\bar{x}$. Its shortcoming is the restricted range of validity: $90^{\circ} \leqslant \varphi \leqslant 180^{\circ}$. In the region $0<\varphi<90^{\circ}$ a "minus" sign must be taken in the right-hand side of [17]. The integration constants arising by solution of the latter equation can be determined by matching it with [22] at the equator. However the matching procedure affects the precision of the result and makes it much worse than Eq. [13] (which is also valid at $\varphi<90^{\circ}$ ).

A closer inspection of Eqs. [10]-[14] reveals that due to the terms with $\operatorname{ctg}(\varphi / 2)$ and $\ln (\sin (\varphi / 2))$ the series for $\bar{x}(\varphi)$ and $\bar{z}(\varphi)$ diverge as $\varphi \rightarrow 0$. This should not be surprising because they represent the outer solution of a singular perturbation problem. Indeed, when the series [6] are substituted in [3] the small parameter $\beta$ turns out to multiply the highest derivative, which is one of the cases when a singular perturbation arises. Therefore, we will now try to find an inner solution (for small $\varphi$ ) by introducing, following the standard procedure $(7,8)$, inner variables:

$$
\tilde{x}=x / \sqrt{\beta}, \quad \tilde{z}=\bar{z}, \quad \tilde{\varphi}=\varphi / \sqrt{\beta} .
$$

We again write the solution as a power series with respect to $\beta$, namely,

$$
\begin{aligned}
& \tilde{x}(\tilde{\varphi}, \beta)=\sum_{n \geqslant 0} \beta^{n} \tilde{x}_{n}(\tilde{\varphi}), \\
& \tilde{z}(\tilde{\varphi}, \beta)=\sum_{n \geqslant 0} \beta^{n} \tilde{z}_{n}(\tilde{\varphi}),
\end{aligned}
$$

where $\tilde{x}_{n}(\tilde{\varphi})$ and $\tilde{z}_{n}(\tilde{\varphi})$ are coefficient functions. Since the series [24] are needed only for $\varphi<5^{\circ}$ (see Section 4), a region of no interest for the system studied by us, we present here only a brief outline of the asymptotic procedure.

Upon substitution of [24] in [3] (written in inner variables), one obtains the following equations for the coefficient functions:

$$
\begin{gathered}
\frac{d \tilde{\varphi}}{d \tilde{x}_{0}}+\frac{\tilde{\varphi}}{\tilde{x}_{0}}=2, \quad \frac{d \tilde{z}_{0}}{d \tilde{\varphi}}=0 \\
\left(\frac{d \tilde{x}_{1}}{d \tilde{x}_{0}}+\frac{\tilde{\varphi}^{2}}{2}\right) \frac{d \tilde{\varphi}}{d x_{0}}+\left(\frac{\tilde{x}_{1}}{\tilde{x}_{0}}+\frac{\tilde{\varphi}^{2}}{6}\right) \frac{\varphi}{\tilde{x}_{0}}=-\tilde{z}_{0},
\end{gathered}
$$

etc. Solution of this system yields

$$
\begin{aligned}
& \tilde{x}_{0}(\tilde{\varphi})=\frac{1}{2}\left(\tilde{\varphi}+\sqrt{\tilde{\varphi}^{2}+4 c_{1}}\right), \quad \tilde{z}_{0}=c_{2}, \\
& \tilde{x}_{1}(\tilde{\varphi})=\frac{\tilde{x}_{0}}{\tilde{x}_{0}^{2}+c_{1}} \\
& \times\left[c_{3}+\frac{c_{1}^{3}}{6 \tilde{x}_{0}^{2}}-\left(1-\frac{c_{1}}{2}\right) \tilde{x}_{0}^{2}-\tilde{x}_{0}^{4} / 6\right],
\end{aligned}
$$

where $c_{1}, c_{2}$, and $c_{3}$ are integration constants to be determined by matching the series [24] with [13] and [14]. The matching procedure yields $c_{1}=2 / 3, c_{2}=2$ and $c_{3}=-8 / 9$. The final results for the inner expansion, written in outer variables, is

$$
\begin{aligned}
& \bar{x}(\varphi)=u(\varphi) \\
& \times\left[1-\frac{27 u^{6}+108 \beta u^{4}+144 \beta^{2} u^{2}-8 \beta^{3}}{54\left(3 u^{4}+2 \beta u^{2}\right)}\right]
\end{aligned}
$$

where

$$
u(\varphi)=\frac{1}{2}\left(\varphi+\sqrt{\varphi^{2}+\frac{8}{3} \beta}\right)
$$


For $\varphi=0$, Eq. [28] yields

$$
\bar{x}(0)=\sqrt{2 \beta / 3}(1-\beta),
$$

where $\bar{x}(0)$ is the abscissa of the extremum of the dashed lines in Fig. 1. Interestingly, if one sets within the parentheses in [30] $\beta=0$, the resulting expression

$$
x(0)=b \sqrt{2 \beta / 3}
$$

(cf. [2]) coincides with the expression of Hartland and Hartley (5) for the radius $r_{\mathrm{c}}$ of the contact line of a bubble of radius $b$ pressed against a flat solid plate. If one introduces the buoyancy force $F_{\mathrm{b}}=\frac{4}{3} \pi b^{3} \Delta \rho g$, Eq. [31] is transformed into the result of Derjaguin and Kussakov (12), $r_{\mathrm{c}}^{2}=b F_{\mathrm{b}} / 2 \pi \sigma$. The latter is a particular case of the more general expression (13) for the radius $r_{\mathrm{c}}$ of the contact line between two bubbles of radii $b_{1}$ and $b_{2}$ and surface tensions $\sigma_{1}$ and $\sigma_{2}$, pressed against each other by a force $F_{\mathrm{b}}$,

$$
r_{\mathrm{c}}^{2}=\bar{b} F_{\mathrm{b}} / 2 \pi \bar{\sigma}
$$

where

$$
\bar{\sigma}=\sigma_{1} \sigma_{2} /\left(\sigma_{1}+\sigma_{2}\right), \quad \bar{b}=b_{1} b_{2} /\left(b_{1}+b_{2}\right) .
$$

Equation [32] was derived in (14) in an alternative way using semiquantitative force balance considerations.. This derivation showed that Eq. [32] (and hence Eq. [31] too) is valid only when the bubble surfaces meet the film, formed between them, at zero contact angle. This result is in agreement with the fact that $\bar{x}(0)$ in [30] has the meaning of the smallest possible radius of contact between a bubble (or drop) and another interface; in other words, if $g \neq 0$, no contact in one point is possible.

\section{PERTURBATIONAL EQUATIONS FOR THE SHAPE OF THE MENISCUS AROUND THE PARTICLE}

The unbounded interface $Z(x)$ [for notations see Fig. 2; it was denoted in Part I by $S(z)$ ] "feels" the particle only through the boundary conditions at the contact line. Therefore, the corresponding solution of the Laplace equation is valid for any axisymmetric

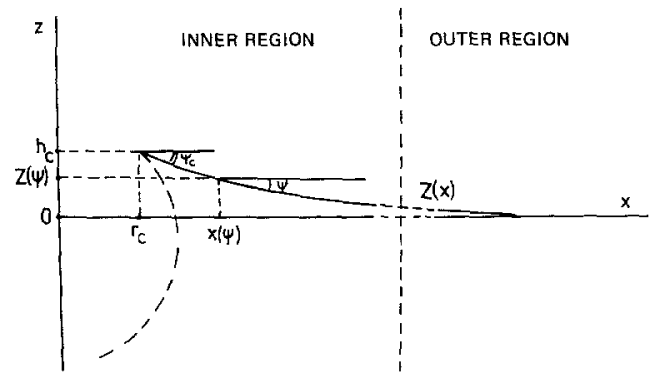

Fig. 2. The external meniscus around an axisymmetric particle (the latter is shown with a dashed line); $r_{\mathrm{c}}$ and $h_{\mathrm{c}}$ are the radius and elevation of the contact line above the horizontal liquid surface and $\psi_{c}$ is the value of the running slope angle $\psi$ at $x=r_{\mathrm{c}}$. The vertical dashed line conventionally separates the inner and outer regions.

subject in contact with this interface, called hereafter the external meniscus.

An approximate solution of this problem was published long ago by Poisson (15) and Rayleigh (16). It was based on the assumption that the slope of the interface is small, and the resulting simplified form of the Laplace equation (this is our Eq. [49]), was assumed uniformly valid for the whole interface. The real situation is, however, different; the slope is small and varies slowly everywhere outside the close vicinity of the particle, but in the latter region it undergoes a sharp increase to the bounding value $\psi_{\mathrm{c}}$. Therefore, we have to deal again with a singular perturbation problem that can be solved efficiently by the method of matched asymptotic expansions $(7,8)$. It is worthwhile noting that well before van Dyke explicitly formulated the above method (7), Derjaguin (17), by making some clever approximations, derived an analytical expression for $h_{\mathrm{c}}$. His procedure is absolutely correct and equivalent to matching the zeroth-order terms of the inner and outer asymptotic expansions. James (18) tried to improve on Derjaguin's result, but his higher order terms are erroneous (see below).

In this section we will derive equations for $h_{\mathrm{c}}$ and $Z(x)$ within terms of the order of $\epsilon^{3}$, where $\epsilon$ is the small parameter (see Eq. [35]). However, since some of the first-order terms 
turn out to be zero and this simplifies many of the following calculations we will at first consider only the terms with $\epsilon^{0}$ and $\epsilon$ and will use these simpler expressions to describe in more detail in Appendix II the matching procedure.

The Laplace equation for the external meniscus reads (9)

$$
\frac{Z^{\prime \prime}}{\left(1+Z^{\prime 2}\right)^{3 / 2}}+\frac{Z^{\prime}}{x\left(1+Z^{\prime 2}\right)^{1 / 2}}=\frac{\Delta \rho g}{\sigma} Z \text {. }
$$

The boundary conditions that the solution of [33] must satisfy are

$$
Z(\infty)=0 ;\left.\quad Z^{\prime}\right|_{r_{\mathrm{c}}}=-\operatorname{tg} \psi_{\mathrm{c}}
$$

Let us introduce the parameters

$$
q=\left(\frac{\Delta \rho g}{\sigma}\right)^{1 / 2}, \quad \epsilon=q r_{\mathrm{c}}
$$

and the outer dimensionless variables (see Fig. 2)

$$
\bar{x}=q x, \quad \bar{Z}=Z / r_{\mathrm{c}}, \quad \chi=h_{\mathrm{c}} / r_{\mathrm{c}} .
$$

The quantity $q^{-1}$ has the dimension of length. The parameter $\epsilon$ is a measure of the ratio of the capillary to the gravity force. For small particles $r_{\mathrm{c}} \ll q^{-1}$, so that $\epsilon \ll 1$. With [35] and [36], Eq. [33] acquires the form

$$
\frac{\bar{Z}^{\prime \prime}}{\left(1+\epsilon^{2} \bar{Z}^{\prime 2}\right)^{3 / 2}}+\frac{\bar{Z}^{\prime}}{\bar{x}\left(1+\epsilon^{2} \bar{Z}^{\prime 2}\right)^{1 / 2}}=\bar{Z}
$$

We look for a solution of [37] as a power series $\bar{Z}(\bar{x}, \epsilon)=\bar{Z}_{0}(\bar{x})+\epsilon \bar{Z}_{1}(\bar{x})+\epsilon^{2} \bar{Z}_{2}(\bar{x})+\cdots$.

This solution is valid only in the outer region and will satisfy only the outer boundary condition $\bar{Z}(\infty)=0$. Therefore, each function $\bar{Z}_{n}$ must satisfy

$$
\bar{Z}_{n}(\infty)=0, \quad n=0,1,2, \cdots .
$$

In the inner region we will replace the independent variable $\bar{x}$ by the inner variable

$$
\xi=\bar{x} / \epsilon
$$

and the dependent variable $\bar{Z}$ will be kept the same. It is more convenient in this case to use the parametric form of the Laplace equation (9). In inner variables Eq. [33] is then replaced by the system of equations

$$
\begin{aligned}
\frac{1}{\xi} \frac{d(\xi \sin \psi)}{d \xi} & =-\epsilon^{2} \bar{Z}, \\
\frac{d \bar{Z}}{d \xi} & =-\operatorname{tg} \psi,
\end{aligned}
$$

where $\psi$ is the slope angle (see Fig. 2). (At the expense of much longer calculations we could have used [37] as basic equation in the inner region as well.) We now seek a solution of the form

$\tilde{Z}(\epsilon \xi, \epsilon)=\tilde{Z}_{0}(\xi)+\epsilon \tilde{Z}_{1}(\xi)+\epsilon^{2} \tilde{Z}_{2}(\xi)+\cdots$,

$\psi(\epsilon \xi, \epsilon)=\psi_{0}(\xi)+\epsilon \psi_{1}(\xi)+\epsilon^{2} \psi_{2}(\xi)+\cdots$.

It must satisfy at $\xi=1$ the inner boundary condition (see [34])

$$
d \tilde{Z} /\left.d \xi\right|_{\xi=1}=-\operatorname{tg} \psi_{\mathrm{c}} \quad \text { or } \quad \psi(1)=\psi_{\mathrm{c}}
$$

which in view of [44] becomes

$\psi_{0}(1)=\psi_{\mathrm{c}} ; \quad \psi_{n}(1)=0, \quad n=1,2,3, \cdots$

By setting $\xi=1$ in [43] we will obtain in view of [36] an expansion for $\chi$,

$$
\chi=\chi_{0}+\epsilon \chi_{1}+\epsilon^{2} \chi_{2}+\cdots,
$$

where

$$
\chi_{n}=\tilde{Z}_{n}(1), \quad n=0,1,2, \cdots
$$

\subsection{Zeroth- and First-Order Terms}

By substituting [38] into [37], expanding in series with respect to $\epsilon$, and setting equal the coefficients before $\epsilon^{n}$ on both sides of the resulting equation, we obtain the following set of equations for the coefficient functions:

$$
\begin{aligned}
& \bar{Z}_{0}^{\prime \prime}+\frac{1}{\bar{x}} \bar{Z}_{0}^{\prime}-\bar{Z}_{0}=0, \\
& \bar{Z}_{1}^{\prime \prime}+\frac{1}{\bar{x}} \bar{Z}_{1}^{\prime}-\bar{Z}_{1}=0,
\end{aligned}
$$


etc. Similarly, Eqs. [41]-[44] lead to

$$
\begin{aligned}
& \frac{1}{\xi} \frac{d\left(\xi \sin \psi_{0}\right)}{d \xi}=0, \frac{d \tilde{Z}_{0}}{d \xi}=-\operatorname{tg} \psi_{0} ; \\
& \frac{1}{\xi} \frac{d\left(\xi \psi_{1} \cos \psi_{0}\right)}{d \xi}=0, \frac{d \tilde{Z}_{1}}{d \xi}=-\psi_{1} / \cos ^{2} \psi_{0} .
\end{aligned}
$$

Equations [49] and [50] are standard Besseltype equations and their solutions are (see also [39])

$$
\bar{Z}_{0}=A K_{0}(\bar{x}) ; \quad \bar{Z}_{1}=B K_{0}(\bar{x}),
$$

where $K_{0}(x)$ is Macdonald's function of the zeroth order [see, e.g., (19)] and $A$ and $B$ are constants to be determined by the matching procedure.

The solutions of [51] and [52] along with the conditions [46] are

$$
\begin{gathered}
\sin \psi_{0}=\frac{c}{\xi} \\
\tilde{Z}_{0}(\xi)=\chi_{0}+c \operatorname{arccosh} \frac{\xi_{c}}{\mathrm{c}}-\mathrm{c} \operatorname{arccosh} \frac{\xi}{c}
\end{gathered}
$$

$$
\psi_{1}(\xi) \equiv 0, \quad \tilde{Z}_{1}(\xi)=\text { const }=\chi_{1},
$$

where

$$
\begin{aligned}
c & =\sin \psi_{\mathrm{c}}, \\
\operatorname{arc} \cosh \xi & \equiv \ln \left(\xi+\sqrt{\xi^{2}-1}\right),
\end{aligned}
$$

and

$$
\xi_{\mathrm{c}}=\bar{x}_{\mathrm{c}} / \epsilon=q r_{\mathrm{c}} / q r_{\mathrm{c}}=1 \text {. }
$$

By substituting [53] in [38] one obtains the two terms of outer expansion, which must be matched with the two terms of inner expansion (following from [54], [55], and [43]) in order to determine the constants $A, B, \chi_{0}$, and $\chi_{1}$. This is done in Appendix II. Here we want to draw the reader's attention only to a subtlety of the procedure. In [54] we have purposely used the notation $\xi_{\mathrm{c}}$ for the boundary value of $\xi$ (although $\xi_{\mathrm{c}}=1$ ), because when going back to outer variables this will no longer be 1 , but will give us terms with $\bar{x}_{\mathrm{c}}$ (see Eq. [58]). On the other hand, because of the scaling used (see Eqs. [35] and [36]) we have $\bar{x}_{\mathrm{c}}=\epsilon$. Nevertheless, when carrying out the expansion in series with respect to $\epsilon$ during the matching procedure, the $\bar{x}_{\mathrm{c}}$, which originates from the boundary conditions, must be treated as a constant.

The matching procedure yields (see Eqs. [84] and [85] in Appendix II)

$$
\begin{aligned}
A & =\sin \psi_{\mathrm{c}}, \quad B=\chi_{1}=0, \\
\chi_{0} & =\sin \psi_{\mathrm{c}} \ln \frac{4}{\gamma_{\mathrm{e}} q r_{\mathrm{c}}\left(1+\cos \psi_{\mathrm{c}}\right)},
\end{aligned}
$$

where $\gamma_{\mathrm{e}}=1.781072418 \cdots$ is Euler's constant. The latter equation exactly coincides with Derjaguin's formula, whose error is therefore of the order of $\epsilon^{2}$; cf. [47] and [59]. As shown by James (18) this formula is also valid within the same accuracy for $\psi_{\mathrm{c}}>90^{\circ}$, i.e., for an interface with a "neck."

\subsection{Second-and Third-Order Terms and Final Results}

In view of [59], Eq. [53] yields $\bar{Z}_{1} \equiv 0$. Then from [37] and [38] one obtains in the outer region

$$
\begin{gathered}
\bar{Z}_{2}^{\prime \prime}+\frac{1}{\bar{x}} \bar{Z}_{2}^{\prime}-\bar{Z}_{2}=\frac{1}{2 \bar{x}} \frac{d}{d \bar{x}}\left(\bar{x} \bar{Z}_{0}^{\prime 3}\right), \\
\bar{Z}_{3}^{\prime \prime}+\frac{1}{\bar{x}} \bar{Z}_{3}^{\prime}-\bar{Z}_{3}=0
\end{gathered}
$$

The solution of [61] for small $\bar{x}$ is

$$
\begin{aligned}
\bar{Z}_{2}(\bar{x})=C \ln \frac{\gamma_{e} \bar{x}}{2} & -\frac{c^{3}}{4}\left(\ln \frac{\gamma_{e} \bar{x}}{2}\right)^{2} \\
& +\frac{c^{3}}{4 \bar{x}^{2}}+\frac{c^{3}}{16}+\cdots
\end{aligned}
$$

The first three terms in the right-hand side of [63] were derived by James (18). The solution of $[62]$ is

$$
\bar{Z}_{3}=D K_{0}(\bar{x})
$$

The integration constants $C$ and $D$ will be determined by the matching procedure. 
The calculations for the second- and thirdorder terms in [43] and [44] are simple but time consuming and therefore we quote only the final results:

$$
\begin{array}{r}
\psi_{2}(\xi) \cos \psi_{0}(\xi)=\frac{c^{3}}{2 \xi}[\Psi(\xi / c)-\Psi(1 / c)], \\
\check{Z}_{2}(\xi)=\chi_{2}+F(\xi / c)-F(1 / c) \\
\psi_{3}(\xi) \equiv 0, \quad \tilde{Z}_{3}(\xi) \equiv \mathrm{const}=\chi_{3},
\end{array}
$$

where

$$
\begin{aligned}
& \Psi(x)=\left(x^{2}-\frac{1}{2}\right) \operatorname{arccosh} x \\
& -\frac{1}{2} x \sqrt{x^{2}-1}-x^{2} \ln \frac{4}{c \gamma_{\mathrm{e}} q r_{\mathrm{c}}} \\
& F(x)=B_{1} x \sqrt{x^{2}-1}+B_{2} \frac{x}{\sqrt{x^{2}-1}} \\
& +\left(B_{3}+\frac{c^{3}}{4} \cdot \frac{2 x-x^{3}}{\sqrt{x^{2}-1}}\right) \operatorname{arccosh} x \\
& +\frac{\mathrm{c}^{3}}{4}\left[x^{2}-(\operatorname{arccosh} x)^{2}\right] \text {; } \\
& \times \ln \left(\operatorname{ctg} \frac{\psi_{c}}{2}\right)+\frac{1}{8} \sin 2 \psi_{c},
\end{aligned}
$$

$$
\begin{aligned}
B_{3}= & \frac{\sin ^{3} \psi_{\mathrm{c}}}{2} \ln \left(\operatorname{ctg} \frac{\psi_{\mathrm{c}}}{2}\right) \\
& -\frac{1}{4}\left(2-3 \sin ^{2} \psi_{\mathrm{c}}\right) \chi_{0}-\frac{1}{8} \sin 2 \psi_{\mathrm{c}} .
\end{aligned}
$$

In these equations $c$ and $\chi_{0}$ are given by [56] and [60], respectively, and the integration constants $\chi_{2}$ and $\chi_{3}$ are to be determined by the matching procedure. It yields $D=\chi_{3}=0$ so that the $\epsilon^{3}$ terms vanish similarly to the $\epsilon$ terms.

The final results for the inner region in dimensional variables $Z$ and $x$ are

$$
\begin{aligned}
\frac{h_{\mathrm{c}}}{r_{\mathrm{c}}} \equiv \chi= & \chi_{0}+\frac{1}{2} \epsilon^{2}\left[\left(\sin \psi_{\mathrm{c}}+\chi_{0} \cos \psi_{\mathrm{c}}\right)\right. \\
& \times\left(1+\chi_{0} \operatorname{ctg} \psi_{\mathrm{c}}-\frac{1}{2} \cos \psi_{\mathrm{c}}\right) \\
& \left.-\frac{1}{2} \chi_{0}+\frac{1}{8} \sin ^{3} \psi_{\mathrm{c}}\right]+O\left(\epsilon^{4}\right),
\end{aligned}
$$

$$
\begin{aligned}
\frac{Z(x)}{r_{\mathrm{c}}}=\frac{h_{\mathrm{c}}}{r_{\mathrm{c}}}-\sin \psi_{\mathrm{c}}\left[\operatorname{arccosh}\left(\frac{x}{r_{\mathrm{c}} \sin \psi_{\mathrm{c}}}\right)\right. \\
\left.-\operatorname{arc~cosh}\left(\frac{1}{\sin \psi_{\mathrm{c}}}\right)\right] \\
+\epsilon^{2}\left[F\left(\frac{x}{r_{\mathrm{c}} \sin \psi_{\mathrm{c}}}\right)-F\left(\frac{1}{\sin \psi_{\mathrm{c}}}\right)\right]+O\left(\epsilon^{4}\right),
\end{aligned}
$$

where the function $F$ is determined by [69][70]. For comparison we quote the expression for $h_{\mathrm{c}} / r_{\mathrm{c}}$ derived by James (18):

$$
\begin{aligned}
& \frac{h_{\mathrm{c}}}{r_{\mathrm{c}}}=\sin \psi_{\mathrm{c}} \ln \left(\frac{4}{\gamma_{\mathrm{e}} q r_{\mathrm{c}}\left(1+\cos \psi_{\mathrm{c}}\right)}\right) \\
& -\frac{\sin ^{3} \psi_{c}}{4} \epsilon^{2}(\ln \epsilon)^{2} .
\end{aligned}
$$

The first term in the right-hand side of [73] coincides with Derjaguin's formula [60]. As James himself admits: "A comparison with numerical data showed that this new estimate for $h_{\mathrm{c}}$ is not an improvement over Derjaguin's formula: the additional term must in fact be positive to increase the accuracy" (18). On the contrary, the leading term (for small angles $\psi_{c}$ ) in the square brackets in our solution [71] is $\left(\chi_{0}^{2} / 2\right) \sin \psi_{\mathrm{c}} \operatorname{ctg}^{2} \psi_{\mathrm{c}}$ and although it also yields formally an $\epsilon^{2}(\ln \epsilon)^{2}$ term, similar to that in [73], its contribution to $h_{\mathrm{c}} / r_{\mathrm{c}}$ is positive. The whole $\epsilon^{2}$ term in [71] is also positive for all angles $0<\psi_{\mathrm{c}}<90^{\circ}$ (except those in the vicinity of $90^{\circ}$ for very small values of $\epsilon$ ). And finally, as the comparison with computer calculations reveals (see next section, Fig. 4) our Eq. [71] is an improvement over Derjaguin's formula. 


\section{NUMERICAL RESULTS AND ESTIMATION OF ERRORS}

In this section we compare the numerical values obtained from our asymptotic solutions with the exact computer calculations of Hartland and Hartley (5) for the bubble surface and Huh and Scriven (4) for the external meniscus.

\subsection{Bubble (Drop) Surface}

In Table I the results for $\bar{x}$ and $\bar{z}$, obtained from [13] and [14] (for sessile interface), are compared with those from (5), $\bar{x}_{\mathbf{H H}}$ and $\bar{z}_{\mathbf{H H}}$, for three values of $\beta$ and two angles $\varphi$. The second angle for each $\beta\left(10\right.$ and $\left.60^{\circ}\right)$ is the limit above which our asymptotic results coincide with the exact values $\bar{x}_{\mathrm{HH}}$ and $\bar{z}_{\mathrm{HH}}$ [note that the authors in (5) use variables different from ours: $X=\bar{x} \beta, Z=\bar{z} \beta$, and $\theta=180^{\circ}$ $-\varphi$ ). It is noteworthy that in spite of formally being of lower order with respect to $\beta$, Eq. [14] has the same precision as [13]. More importantly, the range of applicability of Eqs. [13] and [14] increases with the angle $\varphi$. The latter is also evident from Table II where $\bar{x}\left(90^{\circ}\right)$ and $\bar{z}\left(90^{\circ}\right)$ from [15] and [16] are compared with the exact values $\bar{x}_{\mathrm{HH}}\left(90^{\circ}\right)$ and $\bar{z}_{\mathrm{HH}}\left(90^{\circ}\right)$ and the results obtained from the respective approximate equations of Dorsey $(20) \bar{x}_{\mathrm{D}}\left(90^{\circ}\right)$ and Rayleigh (16) $\bar{x}_{\mathrm{R}}\left(90^{\circ}\right)$ [see also $(5$, p. 650)]. The precision of the solution becomes very high as $\varphi \rightarrow \pi$, because

$$
\lim _{\varphi \rightarrow \pi} \frac{\bar{x}_{1}}{\bar{x}_{0}}=\lim _{\varphi \rightarrow \pi} \frac{\bar{x}_{2}}{\bar{x}_{0}}=\lim _{\varphi \rightarrow \pi} \frac{\bar{z}_{1}}{\bar{z}_{0}}=0
$$

In other words at $\varphi=\pi$ the function $\bar{z}=\bar{z}(\bar{x})$, defined by [13] and [14], is tangent to the circumference $\bar{x}=\sin \varphi, \bar{z}=1+\cos \varphi$. This property of the analytical solution is important, because due to specific computational difficulties the tables of Hartland and Hartley do not contain values for $\varphi>175^{\circ}$.

For $\varphi>15^{\circ}$ the inner solution [28] gave results that were satisfactory, but worse than those obtained from our outer solution [13]. We quote in Table III only the results for $\bar{x}(0)$ from both Eqs. [30] and [31]. It is evident that even at $\beta=10^{-4}$, Eq. [30] is a considerable improvement over Eq. [31].

The above findings are better visualized by the plots of the dependence $\beta(\varphi)$, corresponding to a given constant error of the analytical solution. Since for small $\varphi$ the leading terms in [13] are those with $\operatorname{ctg}(\varphi / 2)$ and $\operatorname{ctg}^{3}(\varphi / 2)$, a natural limit between the regions of "large" and "small" angles is obtained by setting (cf. Eqs. [10] and [11])

$$
\frac{1}{3} \operatorname{ctg} \frac{\varphi}{2} \approx \frac{1}{18} \operatorname{ctg}^{3} \frac{\varphi}{2} \approx 1
$$

This condition is fulfilled for $\varphi \approx 40^{\circ}$. For $\varphi>40^{\circ}$ one has $\bar{x}_{n} \leqq 1(n=0,1,2)$ and one can expect the same to be true for $n \geqq 3$. Let $\Delta \bar{x}=\bar{x}-\left(\bar{x}_{0}+\beta \bar{x}_{1}+\beta^{2} \bar{x}_{2}\right)$, where $\bar{x}_{0}$ $=\sin \varphi$ and $\bar{x}$ is the exact value. If the above assumption holds, $\bar{x}_{3} \approx \bar{x}_{2}$, so that

TABLE I

Numerical Test of Eqs. [13] and [14] against the Tables of Hartland and Hartley (5)

\begin{tabular}{lrllll}
\hline $\log \beta$ & $\begin{array}{c}\varphi \\
\left({ }^{\circ}\right)\end{array}$ & \multicolumn{1}{c}{$\begin{array}{c}\bar{x}_{\mathrm{HH}} \\
(5)\end{array}$} & \multicolumn{1}{c}{$\begin{array}{c}\bar{Z} \\
{[13]}\end{array}$} & $\begin{array}{c}\bar{Z}_{\mathrm{HH}} \\
(5)\end{array}$ & $\begin{array}{c}\bar{Z} \\
{[14]}\end{array}$ \\
\hline-4 & 5 & 0.0879053 & 0.0879052 & 1.99589 & 1.99589 \\
& 10 & 0.174014 & 0.174014 & 1.98455 & 1.98455 \\
-3.6 & 5 & 0.0890142 & 0.0890124 & 1.99543 & 1.99542 \\
& 10 & 0.174564 & 0.174564 & 1.98415 & 1.98415 \\
& 30 & 0.508121 & 0.508100 & 1.8490 & 1.8485 \\
\hline
\end{tabular}


TABLE II

Comparison of Values Predicted by Eqs. [15] and [16] with Those from the Equations of Rayleigh (16) and Dorsey (20) and Computer Calculations (5)

\begin{tabular}{lcccccc}
\hline $\log \beta$ & $\begin{array}{c}\bar{x}\left(90^{\circ}\right) \\
{[15]}\end{array}$ & $\begin{array}{c}\bar{x}_{\mathrm{HH}}\left(90^{\circ}\right) \\
(5)\end{array}$ & $\begin{array}{c}\bar{x}\left(90^{\circ}\right) \\
(16)\end{array}$ & $\begin{array}{c}\bar{x}\left(90^{\circ}\right) \\
(20)\end{array}$ & $\begin{array}{c}\bar{Z}\left(90^{\circ}\right) \\
{[16]}\end{array}$ & $\begin{array}{c}\bar{z}_{\mathrm{HHH}}\left(90^{\circ}\right) \\
(5)\end{array}$ \\
\hline-4 & 0.999983 & 0.999983 & 0.997753 & 1.318646 & 0.999960 & 0.999963 \\
-3 & 0.999833 & 0.999833 & 0.999928 & 1.030388 & 0.999602 & 0.999602 \\
-2 & 0.998342 & 0.998342 & 0.998045 & 1.009856 & 0.996023 & 0.996054 \\
-1 & 0.984211 & 0.984151 & 0.985767 & 0.990834 & 0.960228 & 0.963129 \\
\hline
\end{tabular}

$$
\frac{\Delta \bar{x}}{\bar{x}} \approx \beta^{3} \frac{\bar{x}_{3}}{\bar{x}} \approx \beta^{3} \frac{\bar{x}_{2}(\varphi)}{\bar{x}_{0}(\varphi)} .
$$

If one fixes here the relative error $\Delta \bar{x} / \bar{x}$, one can calculate by means of [11] the curves of constant error $\beta(\varphi)$ for $\varphi>40^{\circ}$.

With small angles, more precisely with $5^{\circ}$ $<\varphi<40^{\circ}$, the values of $\bar{x}_{n}(\varphi)(n=1,2,3)$ are almost entirely determined by the leading terms $\operatorname{ctg}^{2 n-1}(\varphi / 2)$ (see also Appendix I). Therefore we assume $\bar{x}_{3}(\varphi)=$ const $\cdot \operatorname{ctg}^{5}(\varphi)$ 2). Since $\Delta \bar{x} \approx \beta^{3} \bar{x}_{3}$, the constant can be determined by making use of [10], [11], [13], and the exact value of $\bar{x}$ from (5). For several different values of $\beta$ we found const $\approx(2 /$ 15). ${ }^{2}$ Therefore, the curves of constant error can be calculated from the equation

$\frac{\Delta \bar{x}}{\bar{x}}=\frac{2 \cos ^{4}(\varphi / 2)}{225}\left(\frac{\beta}{\sin ^{2}(\varphi / 2)}\right)^{3}, 5^{\circ}<\varphi<40^{\circ}$.

Four curves of constant error $\Delta \bar{x} / \bar{x}=1,0.1$, 0.01 , and $0.001 \%$, calculated from Eqs. [75] and [76], are plotted in Fig. 3. For all points below a given curve the precision of the

\section{TABLE III}

Numerical Test of Eqs. [30] and [31] against Computer Results (5)

\begin{tabular}{|c|c|c|c|}
\hline $\log \beta$ & $\begin{array}{c}\bar{x}(0)_{\mathrm{HH}} \\
(5)\end{array}$ & $\begin{array}{l}\bar{x}(0) \\
{[30]}\end{array}$ & $\begin{array}{l}\bar{x}(0) \\
{[31]}\end{array}$ \\
\hline-4 & $8.16415 \times 10^{-3}$ & $8.16415 \times 10^{-3}$ & $8.16497 \times 10^{-3}$ \\
\hline-3.6 & $1.29373 \times 10^{-3}$ & $1.29373 \times 10^{-2}$ & $1.29406 \times 10^{-2}$ \\
\hline-2 & $8.08481 \times 10^{-2}$ & $8.08332 \times 10^{-2}$ & $8.16497 \times 10^{-2}$ \\
\hline
\end{tabular}

asymptotic solution is higher than that corresponding to the curve. The curves have a small break at $\varphi=40^{\circ}$, which is not surprising in view of the approximate method of error calculation. $\beta$ sharply increases as $\varphi \rightarrow 180^{\circ}$, because then the asymptotic solution tends to

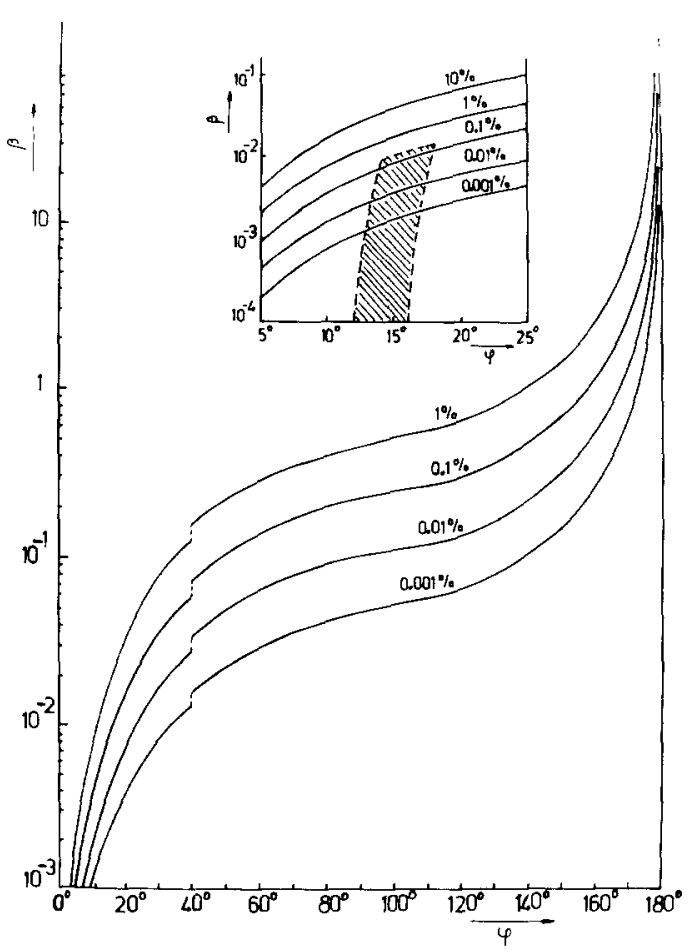

FIG. 3. Curves of constant error $\Delta \bar{x} / \bar{x}$ of Eq. [13]. For all points below a given curve the precision of [13] is higher than that corresponding to the curve (shown in \%). The data for $5^{\circ}<\varphi<25^{\circ}$ are shown on the inset in an enlarged scale; the shaded area indicates the range of values of $\beta$ and $\varphi$ encountered in our experiments (see Part IV). 
the exact shape (see Eq. [74] above). For convenience the inset shows on an enlarged scale only part of the results for $5^{\circ}<\varphi<25^{\circ}$. The shaded area corresponds to the values of $\beta$ and $\varphi$ encountered in our experiments described in Part IV.

\subsection{External Meniscus}

Several curves of constant error $\Delta h_{\mathrm{c}} / h_{\mathrm{c}}$ for the external meniscus for our solution [71] (full lines) and Derjaguin's formula [60] (dashed lines) are shown on Fig. 4. As one could expect Eq. [71] has almost everywhere considerably better precision. The precision of the equation of the meniscus surface [72] is checked in Table IV for $q r_{\mathrm{c}}=\epsilon=0.04159$ and $\psi_{\mathrm{c}}=90^{\circ}$. The first column yields the running slope angle $\psi(x)$. The $Z_{\text {HS }}$ values were taken from the tables of Huh and Scriven (4). Our asymptotic solution intersects the $x$ axis

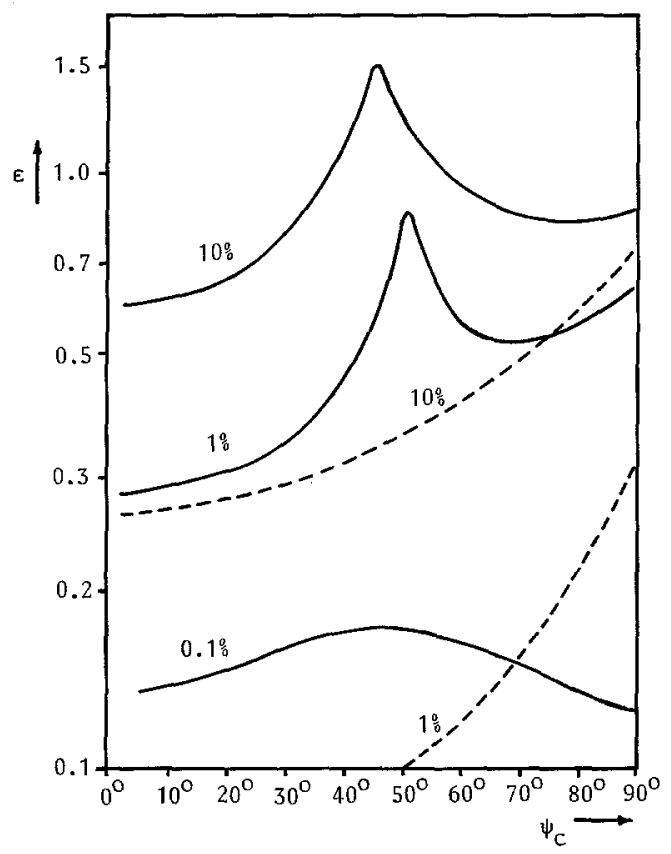

FIG. 4. Curves of constant error $\Delta h_{c} / h_{c}$ of Eq. [71]. For all points below a given curve the precision of Eq. [71] is higher than that corresponding to the curve (shown in \%). The respective curves for Derjaguin's formula [60] are depicted by dashed lines.

\section{TABLE IV}

Numerical Test of Eq. [72] against the Tables of Huh and Scriven (4): $\psi_{\mathrm{c}}=90^{\circ}, \epsilon=q x_{\mathrm{c}}=0.04159$

\begin{tabular}{rccc}
$\begin{array}{c}\psi \\
\left({ }^{\circ}\right)\end{array}$ & $q x$ & $\begin{array}{c}q Z_{\text {Hs }} \\
(4)\end{array}$ & $\begin{array}{c}q Z \\
{[72]}\end{array}$ \\
\hline 80 & 0.04223 & 0.15863 & 0.15862 \\
70 & 0.04424 & 0.15116 & 0.15116 \\
60 & 0.04797 & 0.14320 & 0.14321 \\
50 & 0.05418 & 0.13441 & 0.13441 \\
40 & 0.06444 & 0.12425 & 0.12425 \\
30 & 0.08253 & 0.11177 & 0.11177 \\
20 & 0.11954 & 0.09499 & 0.09499 \\
10 & 0.22701 & 0.06828 & 0.06828 \\
5 & 0.41476 & 0.04519 & 0.04514 \\
2 & 0.81523 & 0.02303 & 0.02250 \\
0.5 & 1.70000 & 0.00690 & 0.00034 \\
- & 1.71600 & - & 0.00000 \\
\hline
\end{tabular}

at $q x=1.71600$ so that close to this value it is no longer valid. Only very far away from the contact line ( $q x \geqq 0.8$ ) does the error of the analytical solution become noticeable. For smaller $\epsilon$, namely, for $\epsilon=0.01012$, the $Z$ values calculated analytically coincide exactly with those of Huh and Scriven for all $\psi_{\mathrm{c}}<90^{\circ}$ (our solution is not valid for $\left.\psi_{\mathrm{c}}>90^{\circ}\right)$. Since for

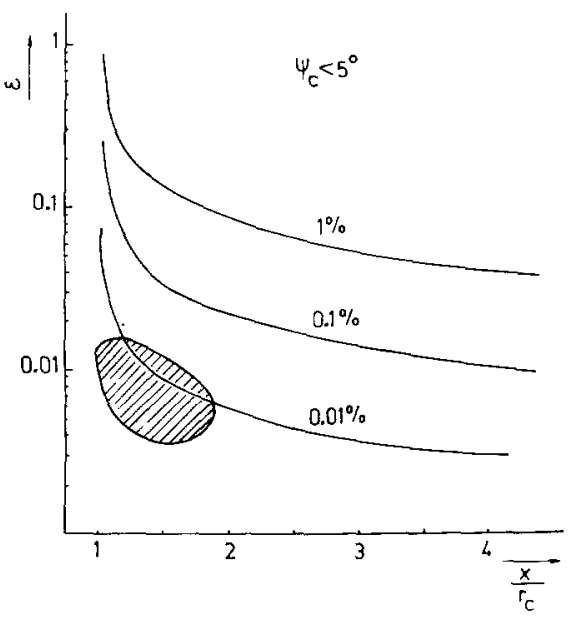

FIG. 5. Relative contribution of $\epsilon^{2}$ term in [72]. Each curve corresponds to a constant ratio of this term to $\left(h_{\mathrm{c}}\right.$ $-Z(x)) / r_{\mathrm{c}}$; this ratio is smaller for the points lying below the respective curve. The shaded area comprises the values of $x / r_{\mathrm{c}}$ and $\mathrm{c}$ encountered in our experiments in Part IV. 
TABLE $V$

True and Apparent Parameters at the Three-Phase Contact Line

\begin{tabular}{cccccccccc}
\hline $\begin{array}{c}c_{c} \\
(\mu \mathrm{m})\end{array}$ & $\begin{array}{c}\boldsymbol{R} \\
(\mu \mathrm{m})\end{array}$ & $\begin{array}{c}b \\
(\mu \mathrm{m})\end{array}$ & $\begin{array}{c}\boldsymbol{R}_{\mathrm{c}} \\
(\mu \mathrm{m})\end{array}$ & $\begin{array}{c}\varphi_{\mathrm{c}} \\
\left({ }^{\circ}\right)\end{array}$ & $\begin{array}{c}\psi_{\mathrm{c}} \\
\left({ }^{\circ}\right)\end{array}$ & $\begin{array}{c}\varphi_{c}^{a} \\
\left({ }^{\circ}\right)\end{array}$ & $\begin{array}{c}\psi_{c}^{a} \\
\left({ }^{\circ}\right)\end{array}$ & $\begin{array}{c}\Delta \varphi_{\mathrm{c}} \\
\left({ }^{\circ}\right)\end{array}$ & $\begin{array}{c}\Delta \psi_{c} \\
\left({ }^{\circ}\right)\end{array}$ \\
\hline 50.9 & 159.8 & 160.0 & 167.3 & 17.72 & 0.93 & 18.57 & 0 & 0.85 & 0.93 \\
41.2 & 158.4 & 158.6 & 169.9 & 14.03 & 1.09 & 15.08 & 0 & 1.05 & 1.09 \\
\hline
\end{tabular}

$\psi_{c}<5^{\circ}$ the function $Z\left(x, \psi_{c}\right)$ turns out to be not sensitive to the variations of $\psi_{\mathfrak{c}}$, it is possible to calculate in this case curves of constant ratio of the $\epsilon^{2}$ term to $(h-z) / r_{\mathrm{c}}$. They are shown in Fig. 5 where the shaded area corresponds to the values of $\epsilon$ and $x / r_{\mathrm{c}}$ observed in our experiments, described in Part IV.

In conclusion we want to warn the reader from trusting too much the "experimental evidence" for the negligible effect of the gravity deformation of the surfaces. This danger is illustrated in Table $\mathrm{V}$ containing the experimentally measured values of $r_{\mathrm{c}}$ and $R$ for two bubbles (for details see Part IV). $R_{\mathrm{c}}$ is the radius of curvature of the generatrix of the bubble surface at the contact line (with $\varphi=\varphi_{\mathrm{c}}$ calculated from our equations). One sees that the deformation occurs mostly near the contact line: $R$ and $b$ are practically equal and $R_{\mathrm{c}}$ differs from them appreciably. If one takes side views of the two bubbles, one can measure only $R$ and $b$ and one could conclude that the bubbles are spherical. One can then calculate apparent values of the angles $\psi_{\mathrm{c}}^{\mathrm{a}}=0$ and $\varphi_{\mathrm{c}}^{\mathrm{a}}$ $=\operatorname{arc} \sin \left(r_{\mathrm{c}} / R\right)$, which will be different from the real values $\psi_{\mathrm{c}}$ and $\varphi_{\mathrm{c}}$. The errors $\Delta \varphi_{\mathrm{c}}=\mid \varphi_{\mathrm{c}}$ $-\varphi_{\mathrm{c}}^{\mathrm{a}} \mid$ and $\Delta \psi_{\mathrm{c}}=\left|\psi_{\mathrm{c}}-\psi_{\mathrm{c}}^{\mathrm{a}}\right|$ (the last two columns in Table V) are well above what is admissible at least for the calculation of film and line tensions (see Part IV).

\section{CONCLUDING REMARKS}

We have derived analytical formulae for the shapes of a sessile (pendant) bubble or drop (Section 2) and an interface (external meniscus) "attached" to a circular ring of radius $r_{c}$, where the slope angle of the interface with re- spect to the horizontal surface is $\psi_{\mathrm{c}}$ (Section 3 ). In both cases we have used asymptotic expansions with respect to a small parameter: $\beta$ $=\Delta \rho g b^{2} / \sigma$ in the former case, and $\epsilon=(\Delta \rho g /$ $\sigma)^{1 / 2} r_{c}$ in the latter case.

The "outer" solution for the bubble is represented by Eqs. [10]-[14] as a parametric function of the coordinates $z$ and $x$ on the running slope angle $\varphi$ (see Fig. 1). It is valid with good precision for $5^{\circ}<\varphi<180^{\circ}$. The "inner" solution, which must be used for $\varphi$ $<5^{\circ}$, is given by Eq. [28], and allows the calculation of the minimum possible radius of contact $x(0)$, Eq. [30]. Another form of the outer solution (for $90^{\circ}<\varphi<180^{\circ}$ ) is represented by Eqs. [19]-[21], yielding the dependence of $\sin \varphi$ on $x$.

The "inner" solution for the shape of the external meniscus $Z(x)$ and for its maximum elevation at the contact line, $h_{\mathrm{c}}$, is given by Eqs. [72] and [71]. Our "inner" solution is compared with that of James (18) and it is shown that the latter is not correct.

In Section 4 our analytical results are checked against the computer calculations of Hartland and Hartley (5) and Huh and Scriven (4). For sufficiently small values of $\beta$ and $\epsilon$ (of the order of those usually encountered in experiments with small bubbles and drops) our solutions proved to be as accurate as the computer calculations.

\section{APPENDIX I}

The Leading TeRm In The SMALL-ANGLE ASYMPTOTICS OF $\bar{x}_{3}$

When $W_{n} \equiv 0$ the first equation [7] is satisfied by $1 / \sin \varphi$. Therefore the general solution 
of the nonhomogeneous equation has the form $\bar{x}_{n}=C_{n}(\varphi) / \sin \varphi$. This yields

$$
\frac{d C_{n}}{d \varphi}=\sin \varphi \cos \varphi W_{n}(\varphi) .
$$

Equations [8] and [10]-[12] show that for small $\varphi, W_{1}(\varphi) \approx-2$ and $W_{2}(\varphi) \sim \varphi^{-4}$. The exact expression for $W_{3}(\varphi)$ is

$$
\begin{aligned}
W_{3}(\varphi)=-\left(\frac{\bar{x}_{1}}{\cos \varphi}\right)^{3} & -\left(\frac{\bar{x}_{1}}{\sin \varphi}\right)^{3} \\
& +2 \frac{\bar{x}_{1} \bar{x}_{2}}{\sin ^{2} \varphi}+2 \frac{\bar{x}_{1} \bar{x}_{2}}{\cos ^{2} \varphi}-\bar{z}_{2}(\varphi) .
\end{aligned}
$$

Therefore, one could expect that $W_{3} \sim \varphi^{-6}$, $C_{3} \sim \varphi^{-4}$, and $\bar{x}_{3} \sim \varphi^{-5}$. Since the leading terms for $\varphi \rightarrow 0$ are $\bar{x}_{1} \sim \operatorname{ctg}(\varphi / 2) \sim \varphi^{-1}$ and $\bar{x}_{2} \sim \operatorname{ctg}^{3}(\varphi / 2) \sim \varphi^{-3}$ one can infer that the leading term in $\bar{x}_{3}$ will be $\operatorname{ctg}^{5}(\varphi / 2)$.

\section{APPENDIX II}

\section{MATCHING OF THE INNER AND OUTER ASYMPTOTIC EXPANSIONS FOR THE EXTERNAL MENISCUS}

According to van Dyke's principle the $m$ terms outer expansion of (the $n$-terms inner expansion) must match the $n$-terms inner expansion of (the $m$-terms outer expansion).

We begin with the two terms expansion from Section 3.1. From [54] and [55] we have for the inner expansion

$$
\begin{aligned}
\tilde{Z}(\epsilon \xi, \epsilon)= & \chi_{0}+c \operatorname{arccosh} \frac{\xi_{c}}{c} \\
& -c \operatorname{arccosh} \frac{\xi}{c}+\epsilon \chi_{1}+O\left(\epsilon^{2}\right)
\end{aligned}
$$

The second and third terms can be rewritten in terms of the outer variable $\bar{x}=\epsilon \xi$ (we recall that according to Eq. [58] $\xi_{\mathrm{c}}$ in outer variables must be replaced by $\bar{x}_{\mathrm{c}} / \epsilon \equiv 1$ )

$$
\begin{aligned}
& \operatorname{arccosh} \frac{\xi_{\mathrm{c}}}{c}-\operatorname{arccosh} \frac{\xi}{c} \\
& =-\ln \frac{\xi+\sqrt{\xi^{2}-c^{2}}}{\xi_{\mathrm{c}}+\sqrt{\xi_{\mathrm{c}}^{2}-c^{2}}}=-\ln \frac{\bar{x}+\sqrt{\bar{x}^{2}-\epsilon^{2} c^{2}}}{\bar{x}_{\mathrm{c}}\left(1+\sqrt{1-c^{2}}\right)} .
\end{aligned}
$$

The latter expression is expanded in series with respect to $\epsilon$,

$$
\begin{array}{r}
-\ln \frac{\bar{x}+\sqrt{\bar{x}^{2}-\epsilon^{2} c^{2}}}{\bar{x}_{\mathrm{c}}\left(1+\cos \psi_{\mathrm{c}}\right)}=-\ln \frac{2}{\bar{x}_{\mathrm{c}}\left(1+\cos \psi_{\mathrm{c}}\right)} \\
-\ln \bar{x}+\frac{1}{4} \epsilon^{2} \frac{c^{2}}{\bar{x}^{2}}+O\left(\epsilon^{4}\right)
\end{array}
$$

where we have also used [56]. By substituting the zeroth-order terms of [78] back into [77] we obtain

$$
\begin{aligned}
& \tilde{Z}(\bar{x}, \epsilon)=\chi_{0}- c \ln \frac{2}{\bar{x}_{c}\left(1+\cos \psi_{c}\right)} \\
&-c \ln \bar{x}+\epsilon \chi_{1}+O\left(\epsilon^{2}\right) .
\end{aligned}
$$

We must now write the two-terms of outer expansion (cf. [53])

$$
\bar{Z}(\bar{x}, \epsilon)=A K_{0}(\bar{x})+\epsilon B K_{0}(\bar{x})+O\left(\epsilon^{2}\right)
$$

in terms of the inner variable $\xi=\bar{x} / \epsilon$, expand it in series with respect to $\epsilon$ (keeping $\xi$ constant), rewrite it back in terms of $\bar{x}$, and retain in the result the zeroth- and first-order terms with respect to $\epsilon$. It is easy to realize that this merely means retaining in [80] the first terms in the expansion of $K_{0}(\bar{x})$ for small $\bar{x}$ [see (19)]:

$\bar{Z}(\bar{x}, \epsilon)=-A \ln \left(\frac{\gamma_{e} \bar{x}}{2}\right)-\epsilon B \ln \left(\frac{\gamma_{e} \bar{x}}{2}\right)+O\left(\epsilon^{2}\right)$.

Since [79] and [81] must match, we thus obtain

$$
\begin{gathered}
\chi_{0}-c \ln \frac{2}{\bar{x}_{\mathrm{c}}\left(1+\cos \psi_{\mathrm{c}}\right)}-c \ln \bar{x} \\
=-A \ln \frac{\gamma_{\mathrm{e}}}{2}-A \ln \bar{x} \\
\chi_{1}=B\left(\ln \frac{2}{\gamma_{\mathrm{e}}}-\ln \bar{x}\right) .
\end{gathered}
$$

Since [82] and [83] must hold identically for any $\bar{x}$, we set (cf. [56])

$$
\begin{aligned}
& A=c=\sin \psi_{\mathrm{c}}, \\
& B=0 ; \quad \chi_{1}=0 .
\end{aligned}
$$


If by means of Eqs. [63]-[67] we calculate the next two terms $\left(\epsilon^{2}\right.$ and $\left.\epsilon^{3}\right)$ in [81] and [79], we will obtain (see also [84] and [85])

$$
\begin{aligned}
\tilde{Z}(\bar{x}, \epsilon) & =c\left[-\ln \frac{\gamma_{\mathrm{e}} \bar{x}}{2}+\left(\frac{\bar{x}}{2}\right)^{2}\left(1-\ln \frac{\gamma_{\mathrm{e}} \bar{x}}{2}\right)\right] \\
+ & \epsilon^{2}\left[\chi_{2}+\frac{c^{3}}{4 \bar{x}^{2}}-\frac{c^{3}}{4}\left(\ln \frac{\gamma_{\mathrm{e}} \bar{x}}{2}\right)^{2}\right. \\
+ & \left.A_{1} \ln \frac{\gamma_{\mathrm{e}} \bar{x}}{2}+A_{2}\right]+\epsilon^{3} \chi_{3}+O\left(\epsilon^{4}\right)
\end{aligned}
$$

where

$$
\begin{aligned}
A_{1}= & \frac{\sin ^{3} \psi_{\mathrm{c}}}{4}\left(1-\cos \psi_{\mathrm{c}}\right)-\frac{\chi_{0}}{4}\left(2-\sin ^{2} \psi_{\mathrm{c}}\right) \\
A_{2}=- & \frac{\sin \psi_{\mathrm{c}}}{2}\left(1+\chi_{0} \operatorname{ctg} \psi_{\mathrm{c}}\right) \\
& \times\left(1+\chi_{0} \operatorname{ctg} \psi_{\mathrm{c}}-\frac{\cos \psi_{\mathrm{c}}}{2}\right)+\frac{\chi_{0}}{4}
\end{aligned}
$$

and

$$
\begin{gathered}
\bar{Z}(\bar{x}, \epsilon)=c\left[-\ln \frac{\gamma_{\mathrm{e}} \bar{x}}{2}+\left(\frac{\bar{x}}{2}\right)^{2}\left(1-\ln \frac{\gamma_{\mathrm{e}} \bar{x}}{2}\right)\right] \\
+\epsilon^{2}\left[\frac{c^{3}}{4 \bar{x}^{2}}-\frac{c^{3}}{4}\left(\ln \frac{\gamma_{\mathrm{e}} \bar{x}}{2}\right)^{2}+C \ln \frac{\gamma_{\mathrm{e}} \bar{x}}{2}+\frac{c^{3}}{16}\right] \\
-\epsilon^{3} D \ln \frac{\gamma_{\mathrm{e}} \bar{x}}{2}+O\left(\epsilon^{4}\right) .
\end{gathered}
$$

The matching of [86] and [88] yields

$$
\begin{aligned}
\chi_{2} & =-A_{2}+\frac{1}{16} c^{3}, \\
C & =A_{1}, \\
\chi_{3} & =D=0,
\end{aligned}
$$

which lead to the final results [71] and [72].

\section{ACKNOWLEDGMENT}

We are indebted to Professor $Z$. Zapryanov for valuable remarks on the asymptotic procedure.

Note added in proof. This paper was already submitted for publication, when we became aware of the work of
L. L. Lo (J. Fluid Mech. 132, 65 (1983)) which is in some respects similar to Section 3 of the present paper. Lo obtained the second order terms in the equation of the external meniscus by using matching in terms of the "inner variables", whereas we do the matching in "outer variables". This difference in procedures has lead to different gauge functions and hence to analytically different results (compare, e.g., her equation [3, 21] with our [71]). Since however both procedures are correct, the numerical results for $h_{c}$ that we obtained from our Eq. [71] and from her Eq. $[3,21]$ were practically the same.

\section{REFERENCES}

1. Ivanov, I. B., Kralchevsky, P. A., and Nikolov, A. D., J. Colloid Interface Sci. 112, 97 (1986).

2. Nikolov, A. D., Kralchevsky, P. A., and Ivanov, I. B., J. Colloid Interface Sci. 112, 122 (1986).

3. Kralchevsky, P. A., Nikolov, A. D., and Ivanov, I. B., J. Colloid Interface Sci. 112, 132 (1986).

4. Huh, C., and Scriven, L. E., J. Colloid Interface Sci. 30, 323 (1969).

5. Hartland, S., and Hartley, R. W., "Axisymmetric Fluid-Liquid Interfaces." Elsevier, Amsterdam, 1976.

6. Padday, J. F., in "Surface and Colloid Science" (E. Matijevic, Ed.), Vol. 1, p. 39. Wiley, New York, 1969.

7. Van Dyke, M., "Perturbation Methods in Fluid Mechanics." Academic Press, New York, 1964.

8. Nayfeh, A. H., "Perturbation Methods." Wiley, New York, 1973.

9. Princen, H. M., in "Surface and Colloid Science" (E. Matijevic and F. R. Eirich, Eds.) Vol. 2, p. 1. Wiley, New York, 1969.

10. Tolman, R. C., J. Chem. Phys. 16, 758 (1948).

11. Buff, F. P., J. Chem. Phys. 19, 1591 (1951).

12. Derjaguin, B. V., Kussakov, M. M., Acta Physicochim. USSR 10, 25 (1939).

13. Ivanov, I. B., Pure Appl. Chem. 52, 1241 (1980).

14. Ivanov, I. B., Dimitrov, D. S., Somasundaran, P., and Jain, R. K., Chem. Eng. Sci. 44, 137 (1985).

15. Poisson, S. D., "Nouvelle Theorie de l'Action Capillaire," Bachelier et Fils, Paris, 1831.

16. Rayleigh, Lord (Strutt, J. W.), Proc. R. Soc. 92, 184 (1915). "Scientific Papers," Vol. 6, pp. 350-361, Dover, New York, 1964.

17. Derjaguin, B., Dokl. Akad. Nauk SSSR 51, 517 (1946).

18. James, D. F., J. Fluid Mech. 63, 657 (1974).

19. Jahnke, E., Emde, F., and Lösch, F., "Tables of Higher Functions." McGraw-Hill, New York, 1960.

20. Dorsey, W. E., J. Wash. Acad. Sci. 18, 505 (1928). 